\title{
Research on Embedded System Platform and Portability Based on ARM
}

\author{
Wu Yong-chong \\ Jiangxi University of Technology,Continuing Education Institute
}

\begin{abstract}
This paper reviews embedded system from a completely new perspective of methodology to elaborate that its nature is technology concept and development approach based on system design. It starts with this concept, and the topic takes platform technology as idea collector of modern design methods to discuss its main composition and realization method and introduce into the field of embedded systems. Base on this, this paper comprehensively constructs a generalized electronic engineering platform stack model and takes its platform or building block and programming models as cores to come up with the infrastructure for embedded system. What's more, this topic takes embedded system with ARM7TDMI CPU core as example to concretely discuss the realization of above infrastructure. In this process, the shortage of automatic migration utility between discovery programming model platform and architecture platform is one of the important reasons for low efficiency of embedded design.
\end{abstract}

Key Words: Embedded system; ARM; Portability; Electronic Engineering

\section{I.INTRODUCTION}

In the information era with continuous integration and science \& technology, antagonism of traditional communication and radar system has become history. Besides, the electronic warfare under high-tech conditions does not only include communication, radar, optical, camouflage, navigation and guidance systems, extending all over space, air, land, surface and underwater, covering meter-wave, microwave, millimeter-wave and all electromagnetic spectrums, involving various arms of service and operation areas. With the increasing demand for electronics, electronic technology gradually becomes the focus of engineering construction, and China also begins to vigorously develop electronic project construction. However, China has a lagging behind basis which has a huge gap comparing to international electronic engineering. In addition, with the continuous development of economic globalization, electronic engineering in China is facing a lot of challenges.

This paper finds out that the shortage of automatic migration utility between discovery programming model platform and architecture platform is one of the important reasons for low efficiency of embedded design. At last, this paper focuses on the portability of embedded platform to create a prototype for source code automatic portable collector, which carries out beneficial practice while improving the automation of embedded system development.

\section{II.TECHNOLOGY OVERVIEW FOR ELECTRONIC ENGINEERING MODERNIZATION}

The internet is developing rapidly which facilitates the expansion of network technology development and makes electronic engineering technology gradually become a relatively independent industry. Nowadays, the network technology is developing rapidly and electronic engineering technology as well as related industries development becomes more and more important. In order to better promote the electronic engineering technology development, better cope with international challenges in the field of electronic engineering technology, we have to timely strengthen the exploration and research on electronic projects.

Electronic engineering is an independent discipline, which carries out overall control and processing on electronic information based on computer and Internet technology. This subject involves electronic equipment, electronic engineering application system development and information processing. Judging by people's dependence level on electronic technology we can find out that electronic technology has affected every aspect of people's lives. In addition, through continuous deepening research on relevant contents, we are able to reduce new 
product development cycle to a certain degree.

At present, the trend for electrical engineering technology business integration and differentiation is gradually obvious, which involves cross-industry development and bringing along with the development and expansion of a series of emerging industries. With the arrival of the knowledge economy, the development for electrical engineering technology has entered a new era. Besides, it has been beckoned as a promising economic type which also promotes the development of local economy. Therefore, continuously strengthening the policy for developing electronics industry with support policies has been an important issue for the government.

\section{III.CHARACTERISTIC PARAMETER FOR EMBEDDED SYSTEM}

In different application fields, there are different requirements on embedded system, which constructs basic specifications. The following parameters can be used to describe the characteristics of embedded system.

Performance:

Generally speaking, it is described based on characteristic frequency. For example, clock speeds or peak workload for Microprocessor, instruction count executed per second (Mega Instructions Per Second, MIPS).

Power Consumption:

Working voltage, working current and drive current of pin terminal all indicate this characteristic.

Reliability:

Continuous work time is commonly used to describe reliability parameters, in addition, adaptation ability to vibrations, humidity, high temperature or low temperature conditions can also demonstrate reliability. Moreover, the number of accessories and complexity of structure can reflect the reliability. The more complicated and accessories are, the worse the reliability, so simple is always better.

Volume:

The size and appearance of embedded system should be in accordance with the volume and appearance of peripheral equipment.

Cost:

The lower cost, the better.

Function:

Functions cannot determine everything because more functions lead to poor reliability and higher cost.

MPU:

The type of CPU is determined by application performance. The lower the MPU frequency and cost is, the better the reliability is. We cannot simply say that 32-bit, $400 \mathrm{MHz}$ ARM is better than 8-bit 12MHz J89C51.

Operation system:

Not all embedded applications require operation system, and it is necessary to load operation system when multiprocessing is needed.

Parameters to describe the above characteristics are known as characteristic parameters which are mutually restricted and should be comprehensively considered. Generally, we start from performance, power consumption, and cost while other parameters are extension of these three parameters.

\section{IV.EMBEDDED DEVELOPMENT PLATFORM BASED ON ARM7TOMI CORE}

This paper takes ARM7TDMI CPU core embedded system as practical case to research on the realization of embedded development platform. Figure 1 demonstrates the view for three-level embedded system. 


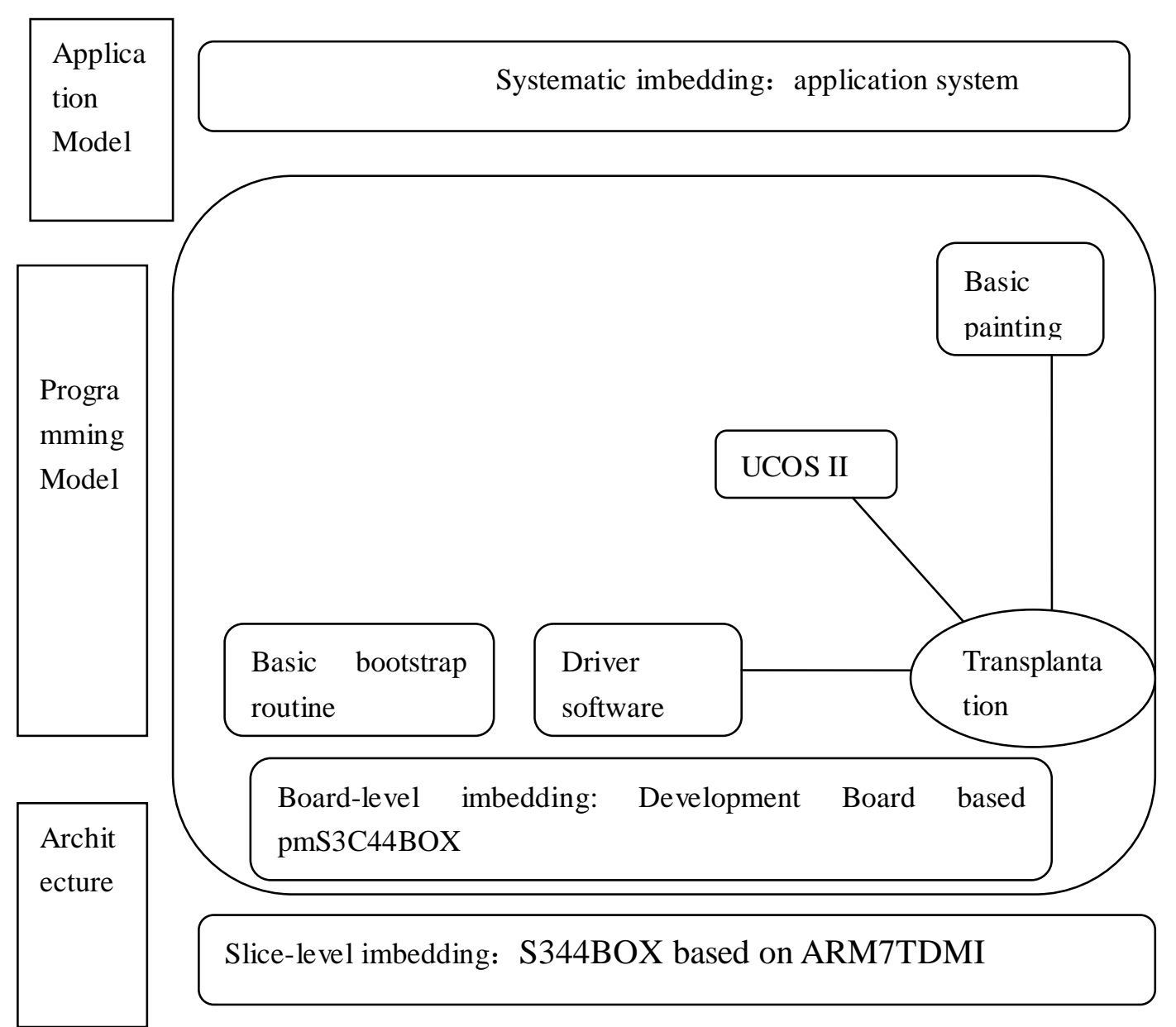

Figure 1. Three-level embedded system

ARM7 series products are developed for consumer applications, including personal audio player, entry-level wireless handheld devices, pagers, inkjet printers, digital cameras, PDA etc, which are sensitive to cost and power consumption. Therefore, ARM7 carries out optimization in these two aspects. ARM7 is developed based on ARM6, ARM6 and previous ARM processors are required to run based on SV power while ARM7 can run below 3V low voltage.

ARM7 series products consist three processor cores: ARM7TDMI, ARM7TDMI-S, and ARM7EJ-S; which also includes a macrocells: ARM720T. The meaning of processor core and macrocells is different because implemented under the guidance of relevant policies, so as to fully take advantage of various social forces, and promote the continuous improvement of China's comprehensive national strength.

The main tasks of this paper include: processor core refers to basic configuration of ARM while macrocells is added with other supporting modules and sometimes we don't emphasize macrocells and adopt unified processor core while readers only have to know that macrocells means processor cores with more functions.

\section{CONCLUSION}

Electronic engineering technology industry has become an important sector in China's economic development, and in order to respond to the Government policy index on developing national economy indicator, electronic engineering technology development can be

1. board module should be debugged such as SD card and USB file management model. Develop corresponding algorithms for DMA data transmission technique and hardware multiplier;

2. The software adopted by this experimental platform should be designed based on system classification with 
corresponding operating instructions so as to promote its use in laboratory teaching for colleges and universities;

3.Peripheral resource of this experimental platform should be further perfected and optimized and carry out relevant design for extension modules.

\section{REFERENCES}

[1]Kuhl M, Reichmann C, Protel I, et al. From Object-Oriented Modeling to Code Generation for Rapid Prototyping of Embedded Electronic Systems $[\mathrm{C}] / /$ Rapid System Prototyping, IEEE International Workshop on. IEEE Computer Society, 2002:108-108.

[2]Raad P E, Komarov P L, Burzo M G. Thermal characterization of embedded electronic features by an integrated system of CCD thermography and self-adaptive numerical modeling[J]. Microelectronics Journal, 2008, 39(7):1008-1015.

[3]Chai Y F, Xiong Y Y, Zhi-Hong W U. Design of embedded electronic bus-stop board based on i.MX53[J]. Computer Engineering \& Design, 2014.

[4]Guo H Z, Chen H, Song T H, et al. CAN Nodes Embedded Automotive Electronic Throttle Simulation System[J]. Journal of System Simulation, 2009, 21(18):5716-5719.
[5]Baronti F, Lenzi F, Roncella R, et al. Embedded electronic control system for continuous self-tuning of motorcycle suspension preload[C]// Control \& Automation, 2007. MED '07. Mediterranean Conference on. IEEE, 2007:1-6.

[6]Guo L X. An Embedded Electronic Police System[J]. Journal of Wuhan Institute of Shipbuilding Technology, 2007.

[7]Zheng H, He Y. Embedded System of Electronic Let-off and Electronic Take-up[J]. Computer Measurement \& Control, 2012, 20(9):2481-491.

[8]Gil L. Optimum functional test method to determine the quality of a software system embedded in a large electronic system: US, US5500941 A[P]. 1996.

[9]Chu Y, Tang K, Chen L, et al. Research of embedded prosthetic control system based on the speech signal[J]. Electronic Design Engineering, 2013.

[10]Xin Q I, Zheng W, Yang G. Real-time Performance Analysis of Embedded Operating System used for Power Electronic System[J]. Power Electronics, 2008.

[11]Feng C, Teng Q, Gui C L. Electronic Control Lubrication Oil SystemBased on Embedded RTOS for Engine[J]. Internal Combustion Engine \& Powerplant, 2007.

[12] Shen H, Zhang J, Li Y, et al. Electronic ticket system based on embedded and RFID technology[J]. Microcomputer \& Its Applications, 2011 